\title{
Development of a 3D numerical wave tank for modeling tsunami generation by underwater landslides
}

\author{
Stéphan T. Grilli ${ }^{\mathrm{a}, 1, *}$, Sylvia Vogelmann ${ }^{\mathrm{a}}$, Philip Watts ${ }^{\mathrm{b}}$ \\ ${ }^{\mathrm{a}}$ Department of Ocean Engineering, University of Rhode Island, Narragansett, RI 02882, USA \\ ${ }^{\mathrm{b}}$ Applied Fluids Engineering, Incorporate, Mail Box \#237, 5710 E. 7th Street, Long Beach, CA 90803, USA
}

Received 23 September 2001; accepted 6 December 2001

\begin{abstract}
A three-dimensional (3D) numerical wave tank (NWT) solving fully nonlinear potential flow theory, with a higher-order boundary element method (BEM), is modified to simulate tsunami generation by underwater landslides. New features are added to the NWT to model underwater landslide geometry and motion and specify corresponding boundary conditions in the BEM model. In particular, a new snake absorbing piston boundary condition is implemented to remove reflection from the onshore and offshore boundaries of the NWT. Model results are favorably compared to recent laboratory experiments. Sensitivity analyses of numerical results to the width and length of the discretization are conducted, to determine optimal numerical parameters. The effect of landslide width on tsunami generated is estimated. Results show that the two-dimensional approximation is applicable when the ratio of landslide width over landslide length is greater than 2. Numerical accuracy is examined and found to be excellent in all cases. (C) 2002 Elsevier Science Ltd. All rights reserved.
\end{abstract}

Keywords: Tsunamis; Landslides; Numerical wave tank; Nonlinear wave transformations; Boundary element method

\section{Introduction}

Tsunamis generated by underwater landslides appear to be one of the major coastal hazards for moderate earthquakes [12,13]. Whereas tsunamis generated by direct coseismic displacement are usually relatively small in height and correlate with moment magnitude, landslide tsunamis are only limited in height by the landslide vertical displacement $[10,15,16]$. Since underwater landslides are usually triggered on the continental slope, their displacement may reach several thousand meters and thus produce huge tsunamis, offering little time for warning due to their proximity to shore $[8,17]$. There is evidence, for instance, that the large tsunami originated near Unimak Island along the Aleutian Trench in 1946 was caused by a giant $\approx 200 \mathrm{~km}^{3}$ underwater landslide, triggered by a magnitude $M_{s}=7.1$ earthquake [2]. The landslide headscarp was on the shallow continental shelf in $150 \mathrm{~m}$ water depth, and the landslide mass moved over a $4^{\circ}$ mean slope, down to the $6000 \mathrm{~m}$ deep Aleutian Terrace, where parts of it apparently stopped. Coastal runup for this tsunami reached $35 \mathrm{~m}$ above sea level at Scotch Cap lighthouse, right onshore of the landslide area.

Predicting landslide tsunamis requires complex numerical models which must accurately represent both landslide and bottom geometry, and the nonlinear interactions between landslide motion and surface wave field. Such a model has been demonstrated by Grilli and Watts [8], in their implementation of a two-dimensional (2D) numerical model for underwater landslides, based on a higher-order boundary element method (BEM), i.e. a numerical wave tank (NWT). ${ }^{2}$ Reviews of the literature to date regarding tsunamis generated by underwater landslides and their numerical modeling can be found in the latter paper and in Watts and Grilli [19].

Here, we describe the current implementation, validation, and simulation of tsunami generation by underwater landslides in the three-dimensional (3D) NWT developed by Grilli et al. [3,4]. Fully nonlinear potential flow equations are solved in this NWT based on a higher-order BEM and an explicit time stepping scheme. Wave overturning can be modeled if it occurs in the computations (Fig. 1). Grilli

\footnotetext{
${ }^{2}$ The concept of NWT was first proposed by Grilli et al. [7] and has
recently blossomed into a broad field of research; see, e.g. Kim et al. [9] for

${ }^{2}$ The concept of NWT was first proposed by Grilli et al. [7] and has
recently blossomed into a broad field of research; see, e.g. Kim et al. [9] for a review of NWTs to date.
}

\footnotetext{
* Corresponding author. Fax: +1-401-874-6837.

E-mail address: grilli@oce.uri.edu (S.T. Grilli).

${ }^{1} \mathrm{http}: / /$ www.oce.uri.edu/ grilli.
} 


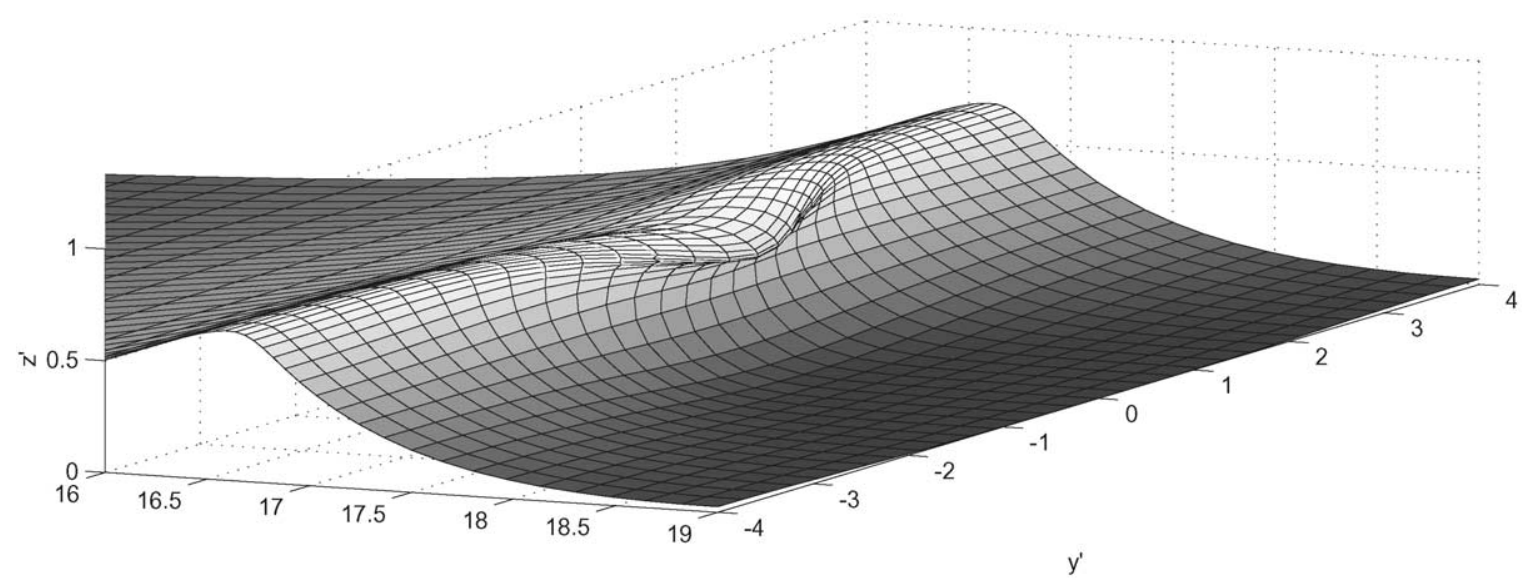

Fig. 1. Example of free surface shape computed for an exact solitary wave of height $H_{0}=0.6 h_{0}$ overturning over a sloping ridge [4].

et al. validated their 3D-NWT for solitary wave shoaling and breaking over slopes, by comparing results both to experiments and to an earlier numerical solution. The agreement was excellent and it was found that a high degree of accuracy could be obtained in the NWT through careful discretization of the simulation domain.

Various improvements were made to this 3D-NWT, as part of the present work, to efficiently and accurately simulate tsunamis caused by underwater landslides. Open boundary conditions were implemented and validated for solitary wave propagation over constant depth. These conditions extend to 3D, the piston-like boundary condition used by Clément [1] and Grilli and Horrillo [5]. The landslide shape and kinematics were modeled on a way similar to Grilli and Watts' [8] 2D model, by assuming a smooth initial shape for the landslide, moving down a planar slope (Fig. 2).

Once the relationship of result accuracy versus spatiotemporal discretization is assessed, numerical experiments can be performed in the 3D-NWT for specified initial and boundary conditions, herein for underwater landslides. This is an important point: the exact nature of wave generation is both known and controlled. Different motions of the same submerged body can produce very different waves, and such waves can be directly related to the input parameters of the motion. This is the basis of the wavemaker formalism introduced by Watts [16,17].

NWTs enable many outputs to be obtained with minimal error, and in virtually no setup time (free surface profiles, numerical wave gages, runup, etc.). Here, however, as done in earlier 2D studies [8], we will usually represent results of the 3D-NWT by a characteristic wave amplitude calculated above the initial landslide position, at the location of maximum landslide thickness (defined at horizontal location $\left(x_{\mathrm{g}}, 0\right)$ in the following; Fig. 4). Our characteristic wave amplitude is thus an implicit function of the underwater landslide shape and motion input parameters.

\section{The numerical wavetank}

\subsection{Governing equations and boundary conditions}

Equations for fully nonlinear potential flows with a free surface, which are solved in the 3D-NWT, are summarized below. The velocity potential, defined as $\phi(\mathbf{x}, t)$, describes inviscid irrotational 3D flows in Cartesian coordinates

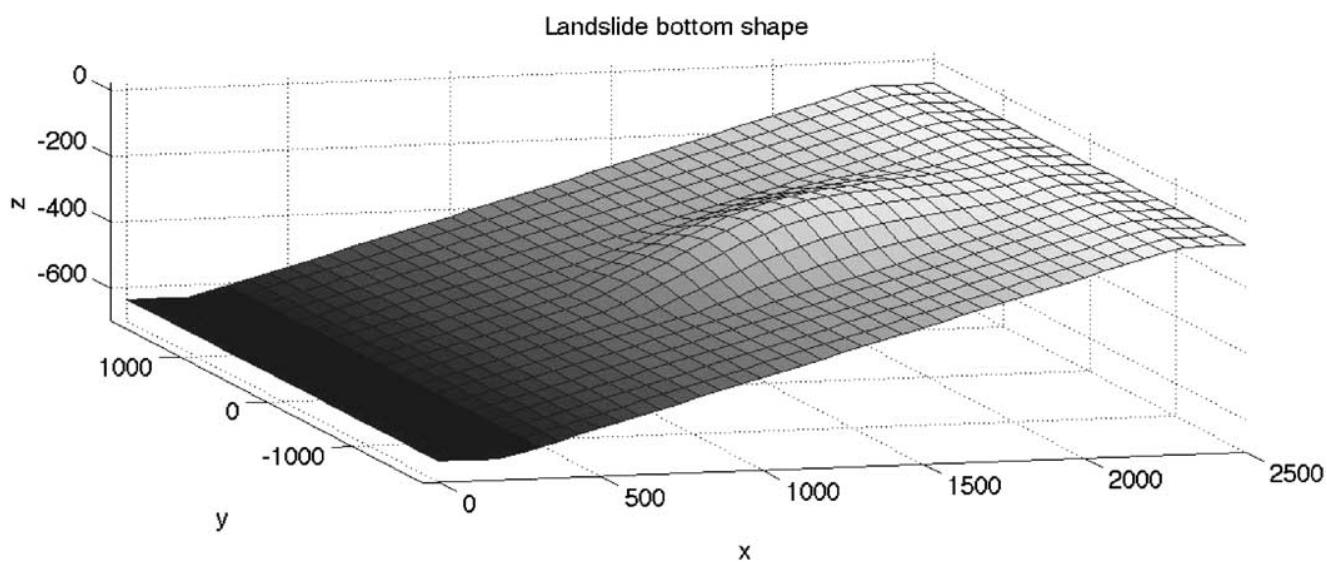

Fig. 2. Example of underwater landslide geometry over a plane $15^{\circ}$ slope, modeled in the 3D-NWT. 


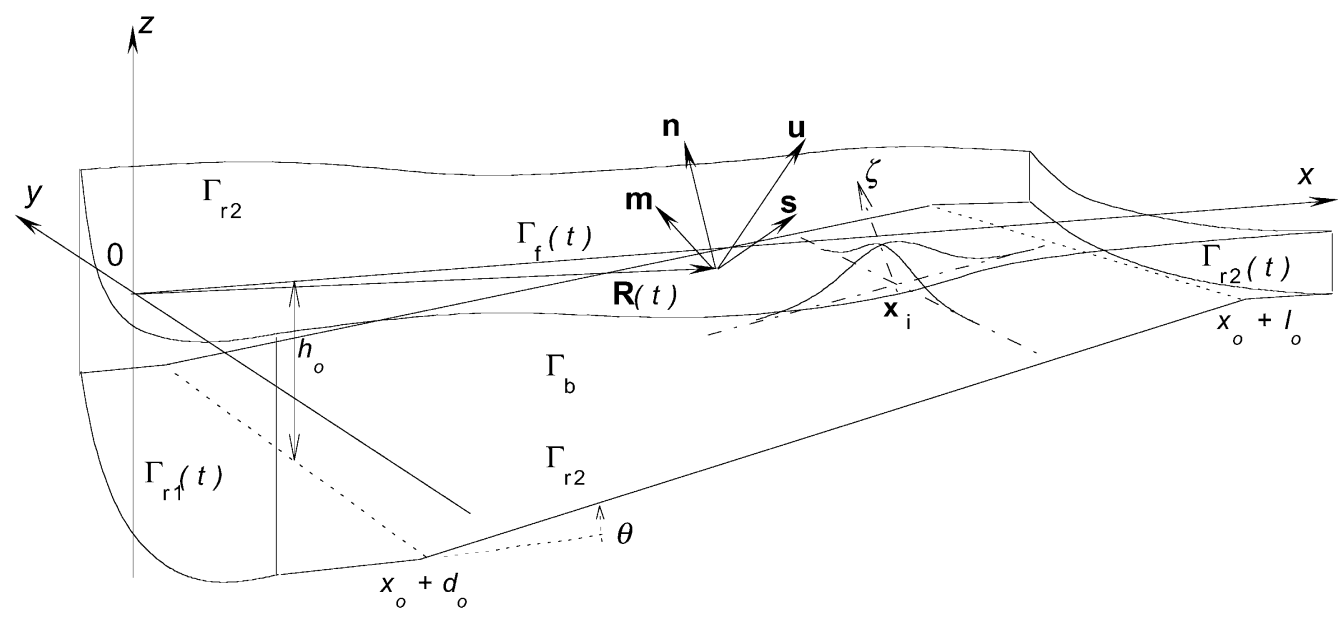

Fig. 3. Sketch of NWT used for landslide tsunami modeling. The landslide is moving in the negative $x$ direction on bottom boundary $\Gamma_{\mathrm{b}}$. Snake absorbing pistons are modeled on boundaries $\Gamma_{\mathrm{r} 1}(t)$ and $\Gamma_{\mathrm{r} 2}(t)$. The bottom is of constant depth $h=h_{0}$ in deep water and sloping from $x=x_{0}+d_{0}$, with slope $\theta$, to $x_{0}+l_{0}$ where a shelf of depth $h_{1}$ of initial length $l_{1}$ is located. The landslide is located on the slope, with its axis intersecting it at point $\mathbf{x}_{i}$ (see also Fig. 2). Tangential vectors at point $\mathbf{R}(t)$ on the free surface $\Gamma_{\mathrm{f}}(t)$ are defined as $(\mathbf{s}, \mathbf{m})$ and outward normal vector as $\mathbf{n}$.

$\mathbf{x}=(x, y, z)$, with $z$ the vertical upward direction (and $z=0$ at the undisturbed free surface; Fig. 3). The velocity is defined by $\mathbf{u}=\boldsymbol{\nabla} \phi=(u, v, w)$.

Continuity in the fluid domain $\Omega(t)$, with boundary $\Gamma(t)$, is a Laplace's equation for the potential

$\nabla^{2} \phi=0 \quad$ in $\Omega(t)$

Green's second identity transforms Eq. (1) into the boundary integral equation (BIE)

$\alpha_{l} \phi_{l}=\int_{\Gamma}\left\{\frac{\partial \phi}{\partial n}(\mathbf{x}) G\left(\mathbf{x}, \mathbf{x}_{l}\right)-\phi(\mathbf{x}) \frac{\partial G}{\partial n}\left(\mathbf{x}, \mathbf{x}_{l}\right)\right\} \mathrm{d} \Gamma$

in which $\alpha_{l}=\alpha\left(\mathbf{x}_{l}\right)=\theta_{l} /(4 \pi)$, with $\theta_{l}$ the exterior solid angle made by the boundary at point $\mathbf{x}_{l}$ (i.e. $2 \pi$ for a smooth boundary), with 3D free space Green's functions defined as

$G=\frac{1}{4 \pi r}$ with $\frac{\partial G}{\partial n}=-\frac{1}{4 \pi} \frac{\mathbf{r} \cdot \mathbf{n}}{r^{3}}$

where $\mathbf{r}=\mathbf{x}-\mathbf{x}_{l}, r=|\mathbf{r}|, \mathbf{x}$ and $\mathbf{x}_{l}=\left(x_{l}, y_{l}, z_{l}\right)$ are points on boundary $\Gamma$, and $\mathbf{n}$ is the outward unit vector normal to the boundary at point $\mathbf{x}$.

The boundary is divided into various sections, with different boundary conditions (Fig. 3). On the free surface $\Gamma_{\mathrm{f}}(t)$, $\phi$ satisfies the nonlinear kinematic and dynamic boundary conditions

$$
\begin{aligned}
& \frac{\mathrm{DR}}{\mathrm{D} t}=\mathbf{u}=\boldsymbol{\nabla} \phi \quad \text { on } \Gamma_{\mathrm{f}}(t) \\
& \frac{\mathrm{D} \phi}{\mathrm{D} t}=-g z+\frac{1}{2} \boldsymbol{\nabla} \phi \cdot \nabla \phi-\frac{p_{\mathrm{a}}}{\rho_{\mathrm{w}}} \quad \text { on } \Gamma_{\mathrm{f}}(t)
\end{aligned}
$$

respectively, with $\mathbf{R}$ as the position vector of a free surface fluid particle, $g$ the acceleration due to gravity, $p_{\mathrm{a}}$ the atmospheric pressure, $\rho_{\mathrm{w}}$ the fluid density, and $\mathrm{D} / \mathrm{D} t$ the material derivative.

Various methods can be used for wave generation in the NWT. Here, tsunamis are generated on the free surface due to a landslide motion $\mathbf{x}_{\ell}(t)$ specified on the bottom boundary $\Gamma_{\mathrm{b}}$ (Fig. 2). We have

$\overline{\mathbf{x}}=\mathbf{x}_{\ell} ; \quad \frac{\overline{\partial \phi}}{\partial n}=\mathbf{u}_{\ell} \cdot \mathbf{n}=\frac{\mathrm{d} \mathbf{x}_{\ell}}{\mathrm{d} t} \cdot \mathbf{n} \quad$ on $\Gamma_{\mathrm{b}}(t)$

where overlines denote specified values, and the time derivative follows the landslide motion. See below for details.

Along stationary parts of the boundary, such as some lateral parts of $\Gamma_{\mathrm{r} 2}$, a no-flow condition is prescribed as

$\frac{\overline{\partial \phi}}{\partial n}=0 \quad$ on $\left(\Gamma_{\mathrm{r} 2}\right),\left(\Gamma_{\mathrm{b}}\right)$

Assuming the landslide motion is in the negative $x$ direction, actively absorbing boundary conditions are specified at one or both extremities of the NWT in the $x$ direction, initially at $x=x_{0}$ and $x_{0}+l_{0}+l_{1}$ (Fig. 3). These are modeled as pressure sensitive 'snake' absorbing piston (AP) wavemakers. The piston normal velocity is specified as

$\frac{\overline{\partial \phi}}{\partial n}=u_{\mathrm{ap}}(\sigma, t) \quad$ on $\Gamma_{\mathrm{r} 2}(t)$

with

$u_{\mathrm{ap}}(\sigma, t)=\frac{1}{\rho_{\mathrm{w}} h_{0} \sqrt{g h_{0}}} \int_{-h_{0}}^{\eta_{\mathrm{ap}}(\sigma, t)} p_{\mathrm{D}}(\sigma, z, t) \mathrm{d} z$

calculated at the curvilinear abscissa $\sigma$, horizontally measured along the piston boundary, where $\eta_{\text {ap }}$ is the surface elevation at the piston and $p_{\mathrm{D}}=-\rho_{\mathrm{w}}\{(\partial \phi / \partial t)+$ $(1 / 2) \nabla \phi \cdot \nabla \phi\}$ denotes the dynamic pressure. The integral in Eq. (9) represents the horizontal hydrodynamic force $F_{\mathrm{D}}(\sigma, t)$ acting on the piston at time $t$, as a function of $\sigma$.

For well-posed problems, we have, $\Gamma \equiv \Gamma_{\mathrm{f}} \cup \Gamma_{\mathrm{b}} \cup$ $\Gamma_{\mathrm{r} 1} \cup \Gamma_{\mathrm{r} 2}$. 


\subsection{Time integration}

Free surface boundary conditions (4) and (5) are integrated at time $t$ to establish both the new position and the boundary conditions on the free surface $\Gamma_{\mathrm{f}}(t)$ at a subsequent time $(t+\Delta t)$ (with $\Delta t$ a varying time step).

To do so, second-order explicit Taylor series expansions are used to express both the new position $\mathbf{R}(t+\Delta t)$ and the potential $\phi(\mathbf{R}(t+\Delta t))$ on the free surface, in an MEL formulation. ${ }^{3}$ The adaptive time step $\Delta t$ in the Taylor series is calculated at each time, from the minimum distance between nodes on the free surface, $\Delta R_{0}$, and a constant mesh Courant number $C_{0}=\Delta t \sqrt{g h_{0}} / \Delta R_{0} \simeq 0.5$ (see Ref. [4] for details).

First-order coefficients in the Taylor series are given by Eqs. (4) and (5), which requires calculating $(\phi,(\partial \phi / \partial n))$ on the free surface. This is done by solving Eq. (2) at time $t$, with boundary conditions (6)-(9). Second-order coefficients are obtained from the material derivative of Eqs. (4) and (5), which requires also calculating $\left((\partial \phi / \partial t),\left(\partial^{2} \phi / \partial t \partial n\right)\right)$ at time $t$. This is done by solving a BIE similar to Eq. (2) for the $\partial \phi / \partial t$ field. The free surface boundary condition for this second BIE is obtained from Bernoulli Equation (4), after solution of the first BIE for $\phi$ as

$$
\frac{\overline{\partial \phi}}{\partial t}=-g z-\frac{1}{2} \nabla \phi \cdot \nabla \phi-\frac{p_{\mathrm{a}}}{\rho_{\mathrm{w}}} \quad \text { on } \Gamma_{\mathrm{f}}(t)
$$

For wave generation by an underwater landslide, Eq. (6) gives

$$
\frac{\overline{\partial^{2} \phi}}{\partial t \partial n}=\frac{\partial\left(\mathbf{u}_{\ell} \cdot \mathbf{n}\right)}{\partial t} \quad \text { on } \Gamma_{\mathrm{b}}(t)
$$

and for stationary boundaries,

$$
\frac{\overline{\partial^{2} \phi}}{\partial t \partial n}=0 \quad \text { on }\left(\Gamma_{\mathrm{r} 2}\right),\left(\Gamma_{\mathrm{b}}\right)
$$

For the absorbing conditions we have, from Eq. (9),

$$
\frac{\overline{\partial^{2} \phi}}{\partial t \partial n}=\frac{\partial u_{\mathrm{ap}}(\sigma, t)}{\partial t} \quad \text { on } \Gamma_{\mathrm{r} 2}(t)
$$

Because of the difference between partial, material, and time derivatives following a boundary motion, it is necessary to further develop Eqs. (11) and (13). For a landslide, we find, from Eq. (11) with Eq. (6) for $\phi_{n}$,

$$
\begin{aligned}
\frac{\overline{\partial^{2} \phi}}{\partial t \partial n}= & \left(\frac{\mathrm{d} \mathbf{u}_{\ell}}{\mathrm{d} t} \cdot \mathbf{n}\right)+\left(\mathbf{u}_{\ell} \cdot \frac{\mathrm{d} \mathbf{n}}{\mathrm{d} t}\right)-\phi_{n} \phi_{n n}-\left(\mathbf{u}_{\ell} \cdot \mathbf{s}\right) \phi_{n s} \\
& -\left(\mathbf{u}_{\ell} \cdot \mathbf{m}\right) \phi_{n m}
\end{aligned}
$$

\footnotetext{
3 This method is a direct extension of that used in the first authors' 2DNWT $[5,6]$. In many 2D studies, it was shown that methods based on explicit second-order Taylor series are more accurate, stable, and efficient (since they accept larger time steps), than implicit Runge-Kutta or predictor-corrector methods.
}

with (indices indicate partial derivatives),

$$
\begin{aligned}
\phi_{n n}= & -\phi_{s s}-\phi_{m m}+\phi_{s}\left\{\mathbf{x}_{s s} \cdot \mathbf{s}-\mathbf{x}_{s m} \cdot \mathbf{m}\right\} \\
& +\phi_{m}\left\{\mathbf{x}_{m m} \cdot \mathbf{m}-\mathbf{x}_{s m} \cdot \mathbf{s}\right\}+\phi_{n}\left\{\mathbf{x}_{s s} \cdot \mathbf{n}+\mathbf{x}_{m m} \cdot \mathbf{n}\right\}
\end{aligned}
$$

where $\mathrm{d} \mathbf{u}_{\ell} / \mathrm{d} t=\dot{\mathbf{u}}_{\ell}$ denotes the absolute landslide acceleration, following landslide motion, and $\mathrm{d} \mathbf{n} / \mathrm{d} t=\dot{\Omega} \mathbf{j} \times \mathbf{n}$ denotes rotation with angular velocity $\dot{\Omega}(y, t)$ around axis $y$ of unit direction $\mathbf{j}$.

From Eq. (13), we find for a snake AP, assuming its motion follows that of water particles,

$\frac{\overline{\partial^{2} \phi}}{\partial t \partial n}=\frac{\mathrm{D} u_{\mathrm{ap}}}{\mathrm{D} t}-\phi_{s} \phi_{n s}-\phi_{m} \phi_{n m}-\phi_{n} \phi_{n n}$

with $\phi_{n n}$ given by Eq. (15). The material derivative of the piston velocity is calculated by backward finite differentiation of Eq. (9). The piston position is calculated for the next time step using a Taylor series expansion similar to the free surfaces

$x_{\text {ap }}(\sigma, t+\Delta t)=x_{\text {ap }}+u_{\text {ap }} n_{x} \Delta t+\frac{\mathrm{D}\left(u_{\mathrm{ap}} n_{x}\right)}{\mathrm{D} t} \frac{(\Delta t)^{2}}{2}$

where the right-hand-side is evaluated at $(\sigma, t)$, and $n_{x}$ denotes the normal vector's $x$-component.

\subsection{BEM discretization}

Definition of the spatial discretization in the NWT follows that of Grilli et al.'s [4] model. All details can be found in the latter reference.

In short, the BIEs for $\phi$ and $\partial \phi / \partial t$ are solved by a BEM. The boundary is discretized into collocation nodes and 2D cubic sliding boundary elements, based on polynomial shape functions. These are expressed over 4 by 4 node reference elements, of which only one 4-node quadrilateral is used as the actual boundary element. Curvilinear changes of variables are used for expressing boundary integrals over reference elements and deriving discretized equations. Discretized boundary integrals, both regular and singular, are calculated for each collocation node by numerical Gauss quadrature (special methods removing singularities, based on polar coordinate transformations in the reference element, are used for the latter). Double and triple nodes and edges are used to specify BIEs at intersecting parts of the boundary [6]. The BEM algebraic system of equations is solved in the present applications using a direct elimination method.

Tangential derivatives, e.g. needed in the Taylor series, are calculated on the boundary in a local curvilinear coordinate system $(\mathbf{s}, \mathbf{m}, \mathbf{n})$ defined at each boundary node (Fig. 3), with $\mathbf{s}=\mathbf{x}_{s}, \mathbf{m}=\mathbf{x}_{m}$, and $\mathbf{n}=\mathbf{s} \times \mathbf{m}$ (subscripts indicate partial derivatives). Derivatives of the geometry and field variables in tangential directions $\mathbf{s}$ and $\mathbf{m}$ are computed, by defining, around each node, a local 5 node by 5 node, fourth-order, sliding element. 


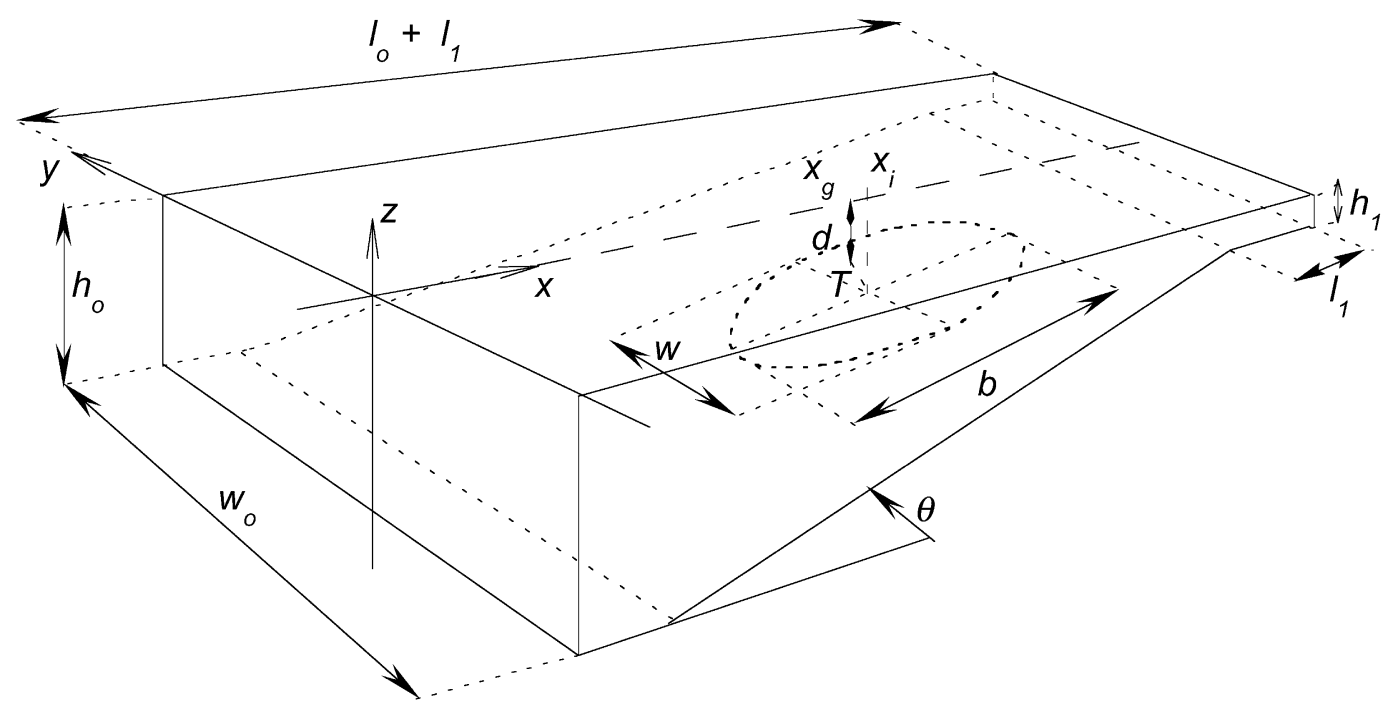

Fig. 4. Definition of landslide initial geometry and corresponding parameters in 3D-NWT.

\subsection{Landslide geometry and discretization}

Semi-ellipses were used to represent the geometry of 2D landslide cross-sections in Grilli and Watts' [8] model. These were moved downslope, according to a specified landslide kinematics. Semi-ellipses, however, introduced sharp corners at the intersection between the landslide and the planar slope, causing singularities in the BEM solution, which required both refining the discretization near corners and rounding out corner geometry [8]. This treatment of corners also required regridding the planar slope nodes both behind and ahead of the landslide, after translating the landslide at each time step.

Here, 3D underwater landslides are represented by a fully submerged smooth sediment mound of density $\rho_{\ell}$, sitting over a plane slope of angle $\theta$ (Figs. 2-4). The landslide has maximum thickness $T$ (measured perpendicularly to the slope). The middle of the landslide surface is located in depth $d$, at a distance $x_{\mathrm{g}}$ along the $x$-axis. Using polar coordinates $\left(r_{\ell}, \varphi_{\ell}\right)$, defined within the slope and centered on the landslide axis intersection with the slope, at point $\mathbf{x}_{i}=$ $\left(x_{i}, y_{i}, z_{i}\right)$, the landslide geometry is represented by $T \operatorname{sech}^{2}\left(k r_{\ell}\right)$ curves, truncated at points where they reach an elevation less than $\varepsilon T$ above the slope. This provides for a smoother bottom geometry than ellipses and eliminates the need for rounding out corners. As a consequence, no bottom regridding is required and the landslide is treated as a 'wave' of bottom elevation moving downslope. The landslide footprint on the bottom slope is defined as an ellipse of major axis $b$ (in the $x$ direction) and minor axis $w$ (in the $y$ direction; Fig. 4). These dimensions are expressed as functions of specified characteristic dimensions $(B, W)$ which, with $T$, define the landslide volume. For the sake of simplicity and comparison with other works, it is assumed that the landslide volume is identical to that of the semi-ellipsoid $(B, W, T)$, i.e.

$V_{\ell}=\frac{1}{6} \pi T B W$

As indicated above, the landslide geometry is defined by its elevation perpendicular to the slope, i.e. (Fig. 3)

$\zeta=\frac{T}{1-\varepsilon}\left\{\operatorname{sech}^{2}\left(k r_{\ell}\right)-\varepsilon\right\}$

where $r_{\ell}=\sqrt{\left(x-x_{i}\right)^{2}+y^{2}}$, with,

$x_{i}=x_{\mathrm{g}}+T \sin \theta ; \quad y_{i}=0 ; \quad z_{i}=d+T \cos \theta$

$x_{\mathrm{g}}=d_{0}+\frac{1}{\tan \theta}\left\{h_{0}-d-\frac{T}{\cos \theta}\right\}$

The ellipse beyond which landslide geometry is truncated is defined as

$r_{\ell 0}\left(\varphi_{\ell}, b, R\right)=\frac{b}{2}\left\{\cos ^{2} \varphi_{\ell}+R^{2} \sin ^{2} \varphi_{\ell}\right\}^{-1 / 2}$

with the ratio $R=b / w=B / W$ and,

$k\left(\varepsilon, \varphi_{\ell}, b, R\right)=\frac{1}{r_{\ell 0}} \operatorname{acosh} \frac{1}{\sqrt{\varepsilon}}$

Equating the landslide volume to $V_{\ell}$ in Eq. (18), we eventually find

$b=B \frac{C \sqrt{1-\varepsilon}}{\left\{3\left[C \sqrt{1-\varepsilon}-\ln \frac{1}{\sqrt{\varepsilon}}-\frac{\varepsilon}{2} C^{2}\right]\right\}^{1 / 2}}$

where $C(\varepsilon)=k r_{\ell 0}=\operatorname{acosh}(1 / \sqrt{\varepsilon})$, and $w=b / R$. In Eq. (23), the term multiplying $B$ is only a function of $\varepsilon$. For $\varepsilon=$ 0.25 , for instance, we find $C=1.317$ and $b=1.371 B$. The 
initial landslide geometry $\mathbf{x}_{\ell}(0)$ is defined using Eqs. (19)(23). An example is given in Fig. 2.

In the NWT, the maximum number of nodes/elements that can be used in the domain discretization is limited by the available computer memory. A tsunami characteristic wavelength was defined by Grilli and Watts [8], based on theoretical scaling considerations as

$\lambda_{0} \simeq t_{0} \sqrt{g d}$

In all cases, the domain length ( $x$ direction) was selected close to that value, and at least $M_{x}=20$ elements were used to discretize one theoretical wavelength.

To provide optimal accuracy in the BEM calculations, the dimensions of the elements should ideally be of similar magnitude along the three dimensions $(x, y, z)$. In the NWT, however, this condition cannot be fully satisfied. The initial horizontal sizes of elements, on the free surface and on the bottom boundaries, can be selected nearly identical in the $x$ and $y$ directions, i.e. $\Delta x_{0} \simeq \Delta y_{0}$. In the vertical direction, however, due to the fact that the same number of elements is used both for the deep water extremity and for the shallow water extremity of the NWT, the element size $\Delta z_{0}$ varies proportionally to $h_{0} / h_{1}$. It is therefore desirable to make the shallow water depth as large as possibly acceptable, with the requirement that all of the landslide be specified over the slope, for the selected $\varepsilon$ and corresponding $b(\varepsilon)$ values. Using the definition of parameters in Fig. 4, this leads to

$h_{1} \leq h_{0}-\left(l_{0}-d_{0}\right) \tan \theta$ with $l_{0} \geq x_{i}-x_{0}+\frac{b}{2} \cos \theta$

Note, however, that using the adaptive integration method developed in Ref. [4], the ratio of longest to shortest elements used in this study reached values of more than 80 , without the BEM solution showing any numerical problem.

Since the modeled landslide geometry is symmetrical with respect to the $y$ axis, this fact is used in some cases to reduce the size of the BEM discretization, by only modeling half of the domain in the $y$ direction, and expressing a no-flow condition along the vertical $(x, z)$ plane. In few cases with very large element size differences along the vertical and horizontal directions around the location of minimum free surface depression, numerical instabilities were observed to develop on the free surface along the plane of symmetry, after a long time of computation. For such cases, no instabilities were observed when the full domain was used instead in the computations (see Ref. [14] for detail).

\subsection{Landslide motion}

We follow the wavemaker formalism developed by Watts [16,17] and used in Grilli and Watts' [8] 2D model, and extend it to 3D. Dimensional analysis shows that, within a family of similar landslide geometry, landslide motion and tsunami characteristics are functions of the five nondimensional independent parameters: $\gamma=\rho_{\ell} / \rho_{\mathrm{w}}, \theta, d / b, T / b$, and $R=w / b$. Watts and Grilli [19] derived an approximate equation of motion describing the center of mass displacement of rigid underwater landslides, $S(t)$, parallel to the planar slope (in the negative $x$ direction). For rigid landslides starting at rest at $t=0$, we have,

$S(t)=S_{0} \ln \left(\cosh \frac{t}{t_{0}}\right)$

with

$S_{0}=\frac{u_{\mathrm{t}}^{2}}{a_{0}} ; \quad t_{0}=\frac{u_{\mathrm{t}}}{a_{0}}$

where $a_{0}$ and $u_{\mathrm{t}}$ denote landslide initial acceleration and terminal velocity, respectively, given by

$a_{0}=g \frac{\gamma-1}{\gamma+C_{\mathrm{m}}} \sin \theta$

where $C_{\mathrm{m}}$ is an approximate added mass coefficient, and

$u_{\mathrm{t}}=\sqrt{g B} \sqrt{\frac{\pi(\gamma-1)}{2 C_{\mathrm{d}}} \sin \theta}$

where $C_{\mathrm{d}}$ is an approximate drag coefficient. Watts [15-17] found added mass and drag coefficients to be of $\mathrm{O}(1)$ for 2D and quasi-2D landslides (for which $W \gg B$ ). (Coulomb friction has been neglected.)

The landslide geometry at time $t$ is found from the initial geometry as

$\mathbf{x}_{\ell}(t)=\mathbf{x}_{\ell}(0)-S(t)(\mathbf{i} \cos \theta+\mathbf{k} \sin \theta)$

which represents a simple translation of the landslide, parallel to the slope, where $\mathbf{i}$ and $\mathbf{k}$ denote unit vectors in the $x$ and $z$ directions, respectively. The center of mass velocity and acceleration at times $t>0$ follow from Eq. (26) as

$\dot{S}(t)=u_{\mathrm{t}} \tanh \frac{t}{t_{0}} ; \quad \ddot{S}(t)=a_{0}\left(\cosh \frac{t}{t_{0}}\right)^{-2}$

Landslide velocity and acceleration at time $t$ are found from Eq. (30) as

$\mathbf{u}_{\ell}=-\dot{S}(t)(\mathbf{i} \cos \theta+\mathbf{k} \sin \theta)$

$\dot{\mathbf{u}}_{\ell}=-\ddot{S}(t)(\mathbf{i} \cos \theta+\mathbf{k} \sin \theta)$

These are used to define bottom boundary conditions (6) and (14).

Fig. 5 shows variations of $S(t), \dot{S}(t)$ and $\ddot{S}(t)$ in nondimensional form, based on the above equations. Because this landslide displacement leads to an impulsive initial acceleration, a ramp-up of the acceleration from 0 to $a_{0>}$ was 


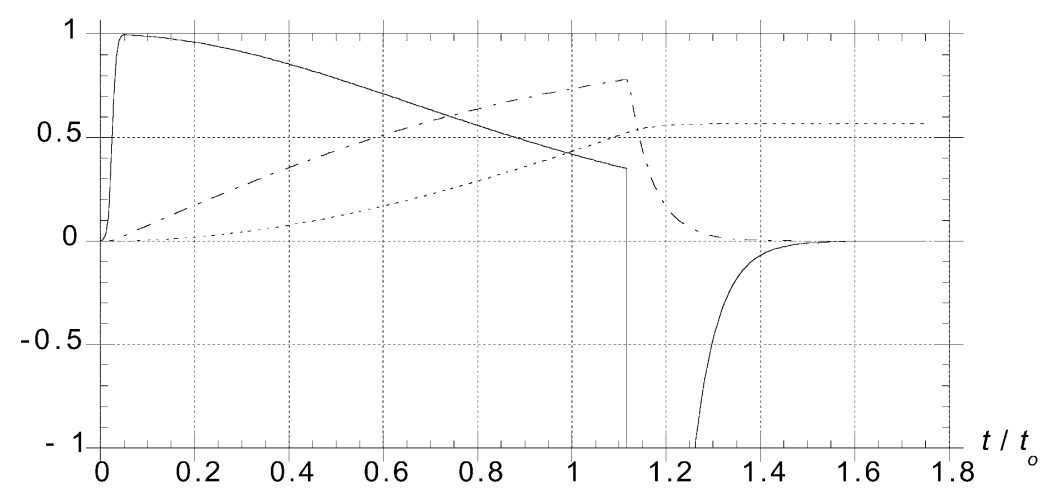

Fig. 5. Nondimensional landslide kinematics modeled as a function of dimensionless time $t / t_{0}: S / S_{0}(---) ; \dot{S} / u_{\mathrm{t}}(---) ; \ddot{S} / a_{0}$. The acceleration ramp-up has a duration $t_{0} / 20$. The sharp deceleration starts at $t=1.15 t_{0}$ (note: the minimum acceleration was truncated for figure scaling purpose).

added to the landslide kinematics model, in the form of a tanh function over a small time interval, $t_{0} / 20$. Also, due to the finite length of the plane slope in the NWT, the landslide is sharply decelerated at some point, in order for the landslide extremity to smoothly reach the slope bottom (at $x=x_{0}+d_{0}$ ) with both a zero speed and acceleration. Equations for the velocity and displacement were modified accordingly. This led to more accurate numerical results in the NWT, and also to a closer approximation of experimental measurements to which model results may be compared [20]. Curves in Fig. 5 reflect this modification.

\section{Applications}

\subsection{Validation of snake AP boundary}

The new AP boundary was validated by propagating a fully nonlinear solitary wave over constant depth $h_{0}$ in the 3D-NWT. The initial wave height is $0.3 h_{0}$ and the initial wave shape, potential, and normal velocity are specified on the free surface based on Tanaka's [11] method. The NWT is $15 h_{0}$ long and discretized with 20 elements over $x$, four elements over $y$ and four elements over $z$. One AP boundary is initially located at the far extremity at $x^{\prime}=x / h_{0}=15$. Due to the 2D geometry and boundary conditions, the AP boundary behaves here as a simple planar piston (within at least six significant figures). Fig. 6 shows vertical cross- sections at $y=0$ in the surface elevations calculated at various times. It is clear that most of the solitary wave leaves the NWT, while only small surface perturbations are left behind. This demonstrates the efficiency of the AP boundary in eliminating (long) wave reflection.

\subsection{Quasi-2D landslide tsunami simulation}

For sake of comparison with earlier 2D results and with recent experimental results, the first landslide application presented here is for a quasi-2D case in which we assume $W \gg B$ and, hence, we do not specify a lateral $(y)$ variation in landslide geometry. Therefore, we model the equivalent of a $2 \mathrm{D}$ slice along a uniform landslide in the 3D-NWT.

We assume the following landslide characteristics: a slope angle $\theta=15^{\circ}$, a rigid landslide with average density $\rho_{\ell}=1860 \mathrm{~kg} / \mathrm{m}^{3}$ and thus $\gamma=1.806$ for $\rho_{\mathrm{w}}=1030 \mathrm{~kg} / \mathrm{m}^{3}$, length $B=1000 \mathrm{~m}$ and maximum thickness $T=52 \mathrm{~m}$ with no lateral variation, and initial submergence $d=261 \mathrm{~m}$. This case was also modeled by Watts et al. [18], using Grilli and Watts [8] 2D-NWT, and results at gage g0 were compared to Watts et al. [20] laboratory experiments, performed at a 1:1000 scale. The landslide model was equipped with an accelerometer, which measured the model acceleration parallel to the slope. Integrating the acceleration twice gave displacement as a function of time. A curve fit of the measurements yielded initial

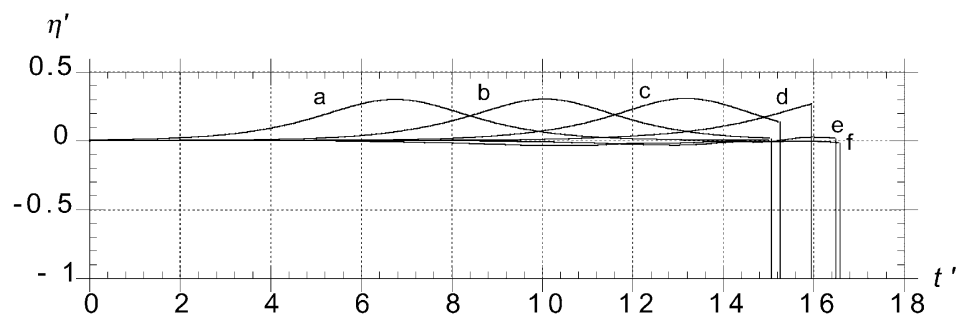

Fig. 6. Dimensionless solitary wave elevation $\eta^{\prime}=\eta / h_{0}$ as a function of $x^{\prime}=x / h_{0}$ computed at dimensionless time $t^{\prime}=t \sqrt{g / h_{0}}=\mathrm{a}: 0$; b: 2.9 ; c: 5.6; d: 8.3; e: 11.5 ; f: 14.9 . 


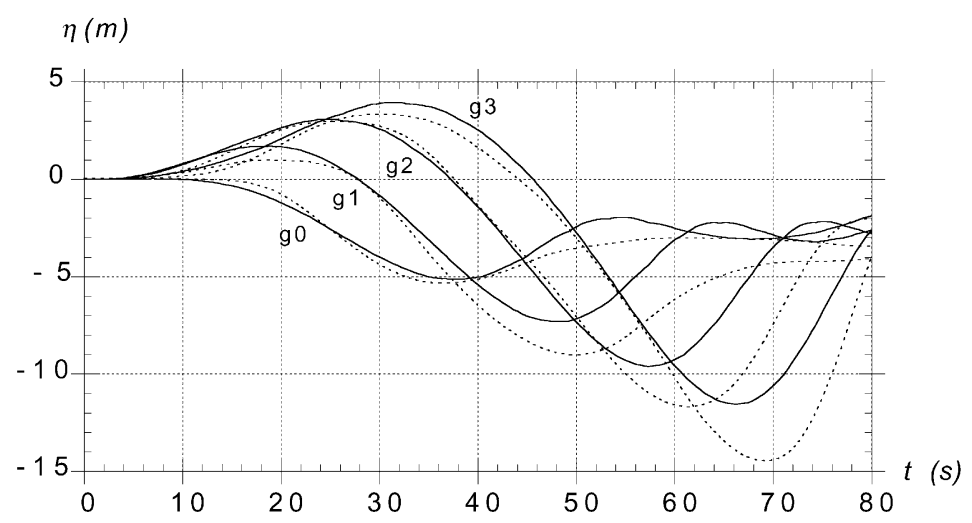

Fig. 7. Landslide tsunami elevations calculated at numerical wave gages $\mathrm{g} 0-\mathrm{g} 3$ (-), as compared to laboratory measurements (- - -) [20], for a quasi-2D case with (prototype scale): $\theta=15^{\circ}, \gamma=1.81, C_{\mathrm{m}}=1.76, C_{\mathrm{d}}=1.53, B=1000 \mathrm{~m}, T=52 \mathrm{~m}, d=261 \mathrm{~m}$. Gages are on the $x$ axis at $x=g 0: 3025.2 \mathrm{~m} ; \mathrm{g} 1$ : $2725.2 \mathrm{~m}$; g2: $2425.2 \mathrm{~m}$; g3: $2125.2 \mathrm{~m}$. Experimental results represent the smoothed average of three replicates of identical experiments.

acceleration $a_{0}$ (following a short duration ramp-up similar to that in Fig. 5) and terminal velocity $u_{\mathrm{t}}$. Parameters $S_{0}$ and $t_{0}$ were obtained from Eqs. (26) and (27), and the added mass and drag coefficients were found from Eqs. (28) and (29), to be $C_{\mathrm{m}}=1.76, C_{\mathrm{d}}=1.53$, respectively. The landslide kinematics was found quite close to that sketched in Fig. 5, with $S_{0}=3673 \mathrm{~m}, u_{\mathrm{t}}=45.91 \mathrm{~m} / \mathrm{s}, a_{0}=0.574 \mathrm{~m} / \mathrm{s}^{2}$, and $t_{0}=80.00 \mathrm{~s}$ (at prototype scale).

The initial NWT domain length, $l_{0}+l_{1}$ (Fig. 4), was selected equal to one approximate tsunami wavelength $\lambda_{0}=4051.0 \mathrm{~m}$ (Eq. (24)). Laboratory experiments were performed using a plane surface piercing slope, i.e. without the shelf seen in Figs. 3 and 4. The shelf, required in the current NWT set-up, was thus assumed to be quite shallow, with $h_{1}=0.075 h_{0}$. The initial shelf length $l_{1}$ was specified such that the volume of water located above the shelf be identical to that of the same triangular section at the top of the slope in the experiments, i.e. $l_{1}=h_{1} /(2 \tan \theta)$. With $x_{0}=0$ and $d_{0}=400 \mathrm{~m}$, we find with this data: $h_{0}=$ $1018.3 \mathrm{~m}, h_{1}=76.37 \mathrm{~m}, l_{0}=3908.5 \mathrm{~m}, l_{1}=142.51 \mathrm{~m}$, $x_{\mathrm{g}}=3025.2 \mathrm{~m}$, and $x_{i}=3038.7 \mathrm{~m}$. In the experiments, the landslide geometry had elliptical cross-sections. In the following results, we used $\varepsilon=0.5$ (and thus $b=1299 \mathrm{~m}$ ), which produces fairly narrow sech ${ }^{2}$ landslide cross-sections, more closely matching an elliptical shape.

In the NWT, we specify 40 BEM elements in the $x$ direction, of initial length $\Delta x_{0}=101.27 \mathrm{~m}$, four elements in the half-width $y$ direction (the minimum required number in the 3D-NWT), of length $\Delta y_{0}=100 \mathrm{~m}$, and six elements over the depth. To better simulate experiments for this quasi-2D case, only one absorbing snake piston boundary is specified in the NWT, at the offshore extremity in the $x$-direction; the onshore vertical boundary is assumed impermeable. ${ }^{4}$ For an

\footnotetext{
${ }^{4}$ Computations performed with a second absorbing boundary led to introducing water into the NWT through the onshore boundary, and to a corresponding increase in water level in the NWT. This may be more physical for actual landslides but does not simulate so well laboratory experiments performed by Watts et al. [20].
}

optimal Courant number of 0.45 [4], the initial time step is set to $\Delta t_{0}=0.45 \Delta x_{0} / \sqrt{g h_{0}}=0.456 \mathrm{~s}$. The total number of nodes is 1054 and there are 848 elements. With these inputs, the CPU time per time step is 3 min $44 \mathrm{~s}$ on a Mac G4$450 \mathrm{MHz}$.

Numerical wave gages were located in the NWT to match the location of capacitance wave gages in the experiments, i.e. $300 \mathrm{~m}$ apart along the $x$ axis, at $x=2125.2,2425.2$, 2725.2 , and $3025.2 \mathrm{~m}$, the latter value being equal to $x_{\mathrm{g}}$. Water elevations calculated at these gages are plotted in Fig. 7, as a function of time, and compared to the scaled-up experimental results. The agreement is quite good, considering the differences in landslide shape and motion, other effects such as sidewall friction, surface tension, and uncertainty on capacitance gage locations, that occur in the experiments, but not in the NWT. The characteristic tsunami amplitude for this case, in the sense of Grilli and Watts [8] and Watts et al. [18,19], is the maximum depression at gage g0, i.e. in Fig. 7, $\left|\eta_{\max }\right|=5.16 \mathrm{~m}$ for the NWT and $5.38 \mathrm{~m}$ for the scaled up experiments.

During these computations, the maximum cumulative numerical error on the domain volume was $0.042 \%$, and the maximum instantaneous error for the dimensionless continuity equation was $0.033 \%$. The maximum error on theoretical landslide volume, due to discretization effects and landslide motion, was $0.40 \%$. It was verified that all of these errors decreased when the size of the discretized elements was also decreased. Results at BEM nodes with different $y$ locations were found identical to within at least six significant figures, which is expected due to the twodimensionality of the problem. This is also the case for the motion of the snake AP boundary, which nearly behaves as a planar piston, as during validation with a solitary wave. Surface elevation and piston velocity for the AP boundary are given in Fig. 8 over a time representing $1.56 t_{0}$. A wave of elevation $5.31 \mathrm{~m}$ high is seen to first leave the NWT through $\Gamma_{\mathrm{r} 1}(t)$, at $t=68.9 \mathrm{~s}$, later followed by a wave of depression $15.57 \mathrm{~m}$ deep. The piston velocity goes from 


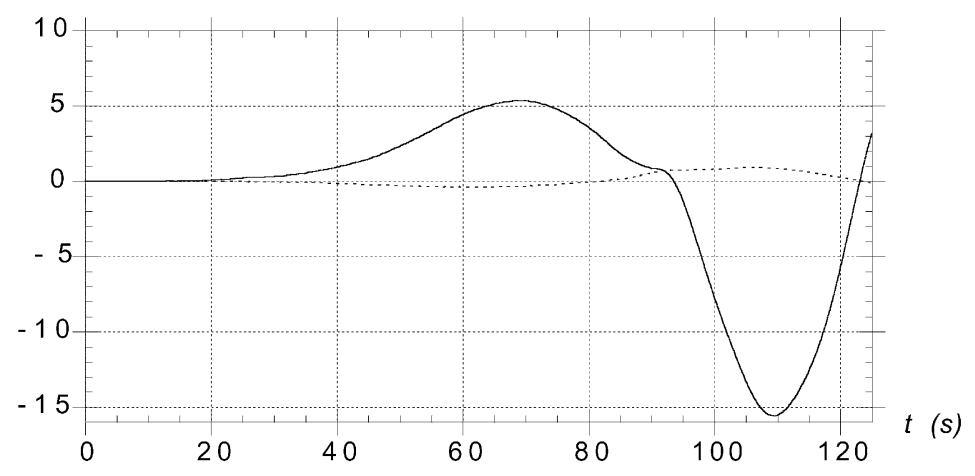

Fig. 8. Same case as Fig. 7. Mean elevation $\bar{\eta}(-)(\mathrm{m})$ and mean piston velocity $\overline{u_{\mathrm{ap}}}(---)(\mathrm{m} / \mathrm{s})$ computed at the offshore AP boundary on $\Gamma_{\mathrm{r} 1}(t)$. Note: the discontinuity in surface elevation around $t=90 \mathrm{~s}$ corresponding to the start of the sharp landslide deceleration in Fig. 5.

negative to positive values and then negative values again, i.e. the piston motion is going away from shore in the early part of the propagation.

\subsection{Three-dimensional tsunami landslide simulations}

We now consider a landslide of similar parameters as in the quasi-2D case above but, this time, there is a lateral $y$ variation in the landslide geometry. Hence, a full 3D landslide geometry is modeled using Eqs. (18)-(23), such as shown in Figs. 2 and 4. We study the sensitivity of results, represented by the sole tsunami characteristic amplitude defined before (i.e. the maximum surface depression at a gage located at $x=x_{\mathrm{g}}$ and $y=0$ ), to the length $L_{0}=l_{0}+$ $l_{1}$ and width $w_{0}$ of the computational domain, and to the landslide width $W$. The sensitivity of results to slope angle $\theta$, landslide length $B$, thickness $T$, and initial submergence $d$, has already been studied by Watts and Grilli [19], using the 2D model by Grilli and Watts [8]. The present 3D study therefore mostly focuses on parameters showing their major influence along the third dimension $y$ (i.e. $w_{0}$ and $w$ ). Finally, only rigid landslides are simulated in this study. Watts and Grilli [19] investigated the influence of landslide deformation, in the form of a linear landslide extension in the downslope direction during failure. They found that the impact of this deformation on the characteristic amplitude was negligible as a first approximation.

The computational domain is such as sketched in Fig. 4. The offshore boundary is represented by an AP boundary and all other lateral boundaries are assumed impermeable. The landslide kinematics is computed based on Eqs. (26)(32). The shallow shelf at the onshore extremity has depth $h_{1}=70 \mathrm{~m}$ and length $l_{1}=300 \mathrm{~m}$. The constant depth offshore region has a length $d_{0}=400 \mathrm{~m}$ (with $x_{0}=0$ ), and its depth $h_{0}=h_{1}+l_{0} \tan \theta$ depends on the selected domain length. The landslide parameters that will be maintained constant throughout this study are slope angle $\theta=$ $15^{\circ}$, rigid landslide with average density $\rho_{\ell}=1900 \mathrm{~kg} / \mathrm{m}^{3}$ (and thus $\gamma=1.845$ for $\rho_{\mathrm{w}}=1030 \mathrm{~kg} / \mathrm{m}^{3}$ ), landslide length $B=1000 \mathrm{~m}$ (and thus $b=1299.1$ for $\varepsilon=0.5$ ), maximum landslide thickness $T=50 \mathrm{~m}$ and initial submergence $d=$ $260 \mathrm{~m}$. The hydrodynamic coefficients are set to the standard values $C_{\mathrm{m}}=1, C_{\mathrm{d}}=1$. With this data, the landslide kinematics is defined by $S_{0}=4469 \mathrm{~m}, u_{\mathrm{t}}=58.1 \mathrm{~m} / \mathrm{s}, a_{0}=$ $0.754 \mathrm{~m} / \mathrm{s}^{2}$, and $t_{0}=77.0 \mathrm{~s}$, and, based on this, we find an approximate tsunami wavelength $\lambda_{0}=3888 \mathrm{~m}$.

For a domain length $L_{0}=\lambda_{0}$, with $h_{0}=1031.4 \mathrm{~m}, W=$ $B$, and $w_{0}=2 W$, we specify $20 \mathrm{BEM}$ elements in the $x$ direction, of initial length $\Delta x_{0}=194.4 \mathrm{~m}, 10$ elements in the width $y$ direction, of length $\Delta y_{0}=200 \mathrm{~m}$, and four elements over depth. The initial time step is set to $\Delta t_{0}=$ $0.45 \Delta x_{0} / \sqrt{g h_{0}}=0.87 \mathrm{~s}$. Fig. 9 shows free surface elevations computed in this simulation at various times $t=0$ to $31.6 \mathrm{~s}$. We see the appearance of an initial dipole-like surface elevation, with a wave of depression forming above the landslide initial location and a wave of elevation propagating offshore of the landslide. Later on $(t>21 \mathrm{~s})$, a smaller wave of elevation is seen to also propagate onshore, which would eventually induce coastal flooding and runup. For this case, the maximum error on mass conservation was $0.82 \%$ of the landslide volume.

We maintain the same density of discretization for other cases studied in the sensitivity analysis, using different domain length and width, and landslide width. Results are presented as follows.

\subsubsection{Effect of domain length}

For simplicity, quasi-2D simulations, i.e. cases with no variation in the $y$-direction $(w=\infty)$, are modeled in order to investigate the influence of the total domain length on tsunami characteristic amplitude. The domain width is set to $w_{0}=B$ for all calculations.

The total domain length $L_{0}$ is set to multiples of the theoretical wavelength $\lambda_{0}(0.6-3)$. As indicated before, a constant grid density of 20 elements per theoretical wavelength is used in the $x$-direction, which provides the same BEM resolution and accuracy for all cases. Results are shown in Fig. 10. The characteristic amplitude for fully developed quasi-2D tsunami generation is seen to be approximately $6.0 \mathrm{~m}$. For $L_{0}>\lambda_{0}$, little change can be 

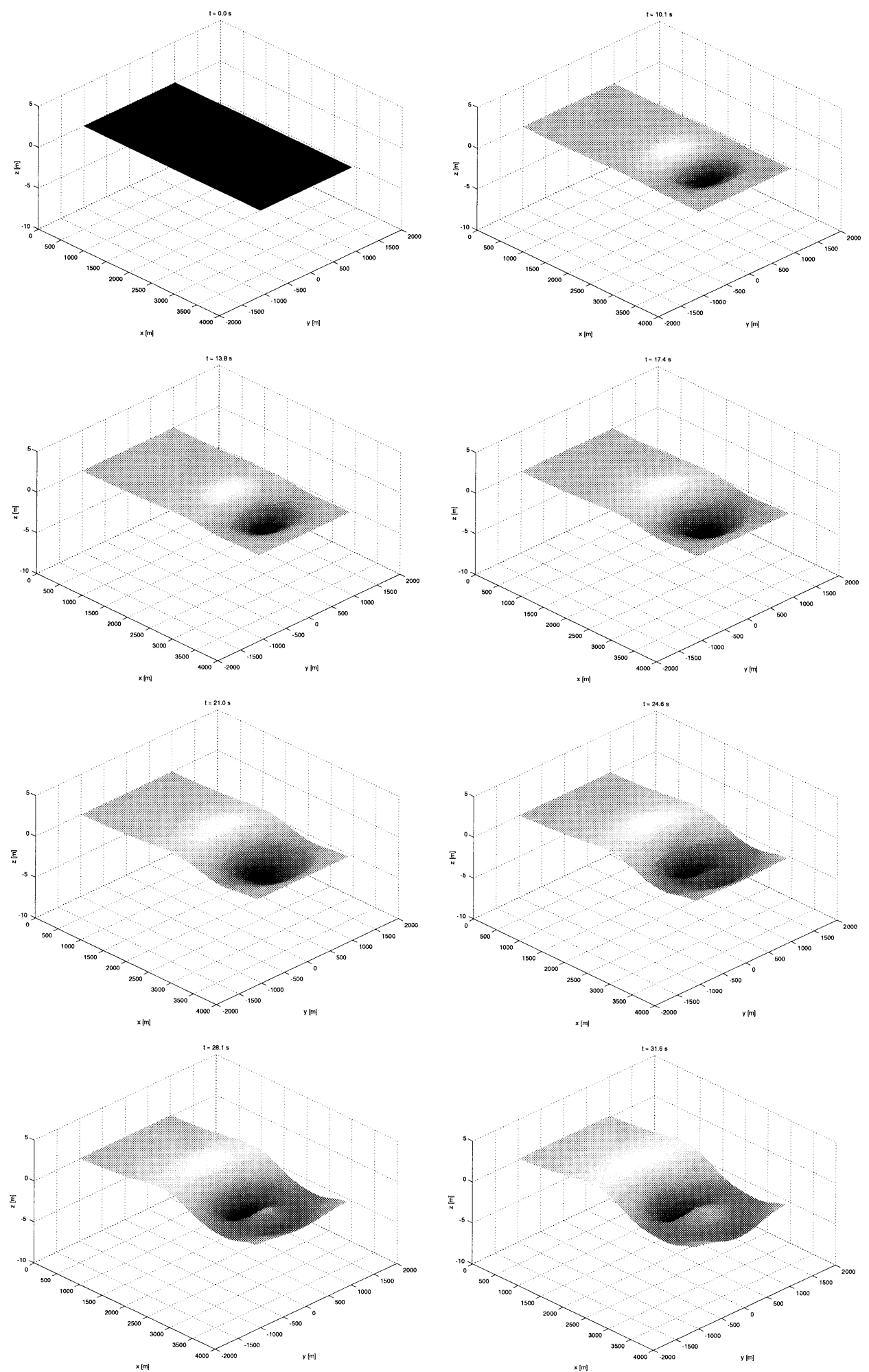

Fig. 9. Surface elevation at different times for a 3D landslide tsunami simulation with $\theta=15^{\circ}, B=1000 \mathrm{~m}, W=1000 \mathrm{~m}, T=50 \mathrm{~m}, d=260 \mathrm{~m}, L_{0}=$ $l_{0}+l_{1}=\lambda_{0}=3888 \mathrm{~m}, w_{0}=2000 \mathrm{~m}, d_{0}=400 \mathrm{~m}, h_{0}=1031.4 \mathrm{~m}, l_{1}=300.0 \mathrm{~m}, h_{1}=70.0 \mathrm{~m}, C_{\mathrm{m}}=1, C_{\mathrm{d}}=1$. 


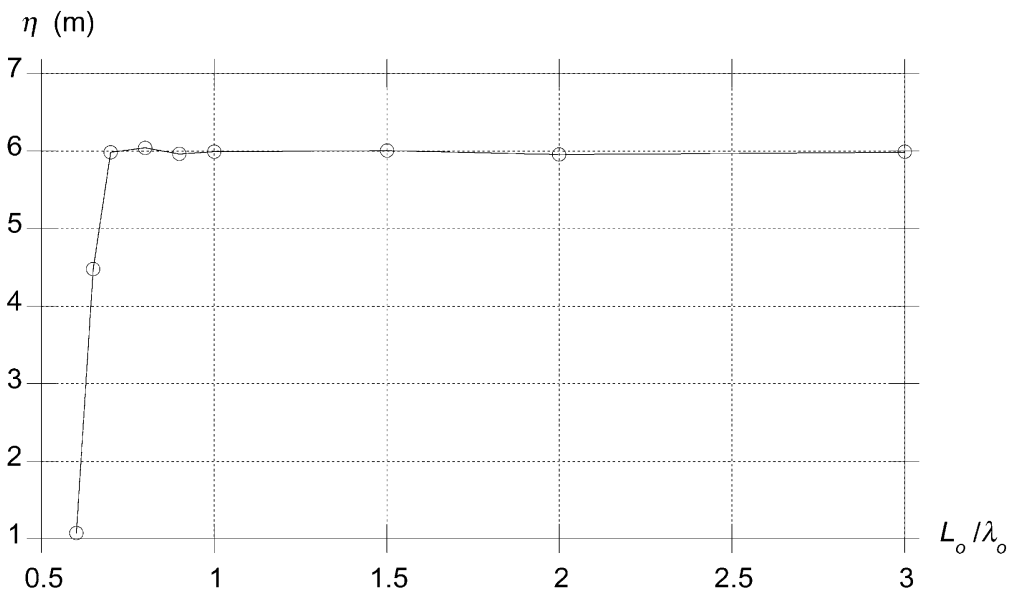

Fig. 10. Characteristic tsunami amplitude for quasi-2D simulations with varying ratios of domain length over theoretical wavelength $L_{0} / \lambda_{0}$, with $w=\infty$ and $w_{0}=B$.

seen in the computed characteristic amplitude. Hence, this value will be used for domain length in the following.

The maximum change in domain volume in these computations is $0.06 \%$, and the corresponding value with respect to the landslide volume is $0.65 \%$. These values are quite small and therefore ensure high accuracy of the calculations.

\subsubsection{Effect of domain width}

When performing numerical calculations, it is desirable to minimize the influence of numerical parameters on results. In particular, here, it is important to verify at which distance, $y= \pm w_{0} / 2$, the impermeable lateral boundaries have to be located to avoid perturbing the tsunami generation process, i.e. changing the characteristic tsunami amplitude, through reflection, while maintaining a realistic size for the computational domain.

To do so, landslides of different widths $W=B, 2 B$ and $3 B$ are modeled and, for each width, calculations are performed using different domain widths $w_{0}=W$ to $3-6 W$. The total length of the domain is maintained equal to the theoretical wavelength, $L_{0}=\lambda_{0}$, and the resolution of the BEM discretization is similar to above. Fig. 11 shows results of computations. We see that, for a ratio $w_{0} / W \geq 2$, the characteristic amplitude only varies very slightly. Hence, this ratio will be used in the following.

\subsubsection{Effect of landslide width}

Landslides of various widths $W=B / 2$ to $3 B$ are modeled and the resulting characteristic amplitudes compared. According to the above findings, calculations use $w_{0}=$ $2 W$ and $L_{0}=\lambda_{0}$. Fig. 12 shows results of these computations. Essentially, we see an increase in characteristic amplitude with increasing landslide width, i.e. landslide aspect ratio $W / B$. The large initial increase with $W / B$, however, becomes slower for $W / B>2$, where the amplitude eventually approaches its $2 \mathrm{D}$ value of $\simeq 6 \mathrm{~m}$ asymptotically.

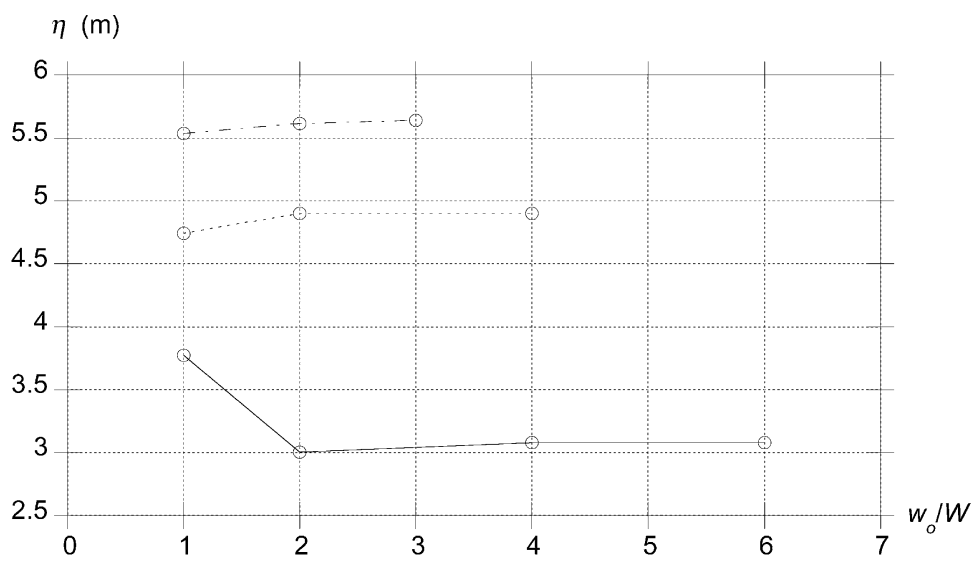

Fig. 11. Characteristic tsunami amplitude for varying domain widths $w_{0}$, with $L_{0}=\lambda_{0}$ and $W=(-) B$; (- - - ) $2 B$; and (- - $) 3 B$. 


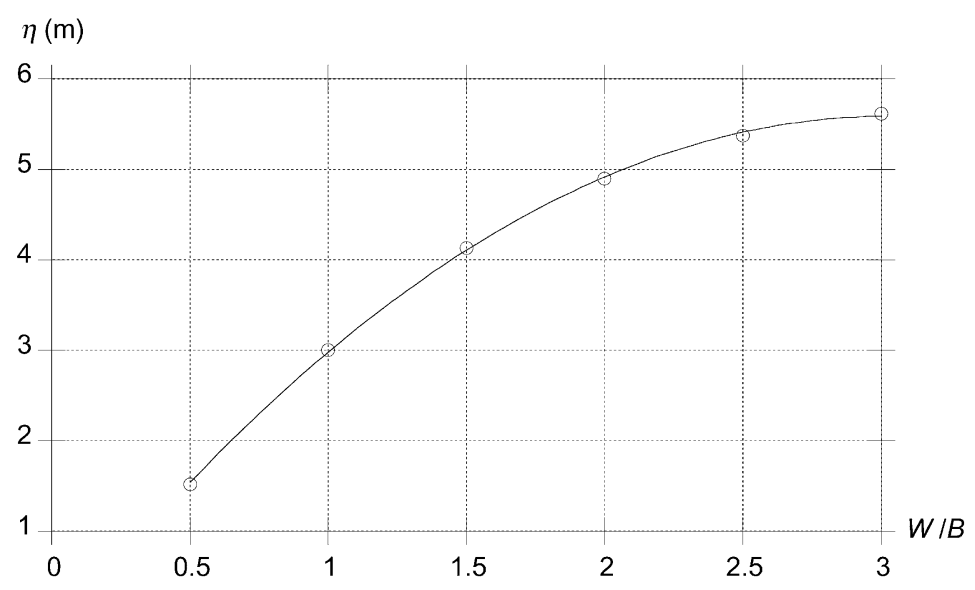

Fig. 12. Characteristic tsunami amplitude as a function of landslide aspect ratio width over length $W / B$, with $L_{0}=\lambda_{0}$, and $w_{0} / W=2$. (O) computations; ( - ) curve fit $\eta=-0.217+3.83(W / B)-0.632(W / B)^{2}\left(R^{2}=0.999\right)$.

\section{Conclusions}

Realistic simulations of tsunami generation by submarine landslides can be obtained by performing wave tank experiments. However, a well-validated numerical model can often provide as accurate or even more accurate results, in some cases, than experiments, in a much shorter time. This is important for assessing tsunami hazard in specific situations.

Here, landslide tsunami generation mechanisms were explored in a 3D NWT solving fully nonlinear potential flow equations, using a higher-order BEM. To do so, new features were added to the NWT (model of underwater landslide geometry and motion, snake AP boundaries) and their accuracy and efficiency was tested in some applications.

Numerical results in the 3D-NWT were validated by comparing computed surface elevations to laboratory measurements for a quasi-2D landslide. The agreement was quite good, particularly for the characteristic tsunami amplitude. Other similar comparisons will be presented in future work, for fully 3D landslide experiments [20].

Based on a sensitivity analysis carried out for realistic landslide parameter values, we conclude that the characteristic tsunami amplitude is not significantly affected when the width of the computational domain $w_{0} \geq 2 \mathrm{~W}$ and the length of the computational domain $L_{0} \geq \lambda_{0}$, respectively. We also find that the 3D characteristic tsunami amplitude converges towards the $2 \mathrm{D}$ value for $W \geq 2 B$. The latter result is of importance as $2 \mathrm{D}$ analyses (such as described in Ref. [8]) are much easier and quicker to carry out than 3D studies, particularly in a realtime forecasting situation. It is therefore of interest to assess whether 2D conditions can be assumed in a given case and the corresponding simplified model applied.

The numerical results found in this sensitivity analysis are not strictly general, since they were derived for a single slope and a constant landslide density and length. It is expected, however, that the same qualitative findings regarding the effects of $L_{0}, w_{0}$ and $w$, on characteristic tsunami amplitude, would apply to other landslides, albeit with some differences in the numerical results. Such differences could then be estimated using the empirical functions derived by Watts and Grilli [19].

To our knowledge, fully nonlinear 3D wave computations have never been reported for landslide tsunami sources. Such numerical wave fields could be used as realistic initial conditions in long-wave-based tsunami propagation and runup models.

\section{Acknowledgements}

This work was supported by Grant CMS0100223 of the US National Science Foundation.

\section{References}

[1] Clément A. Coupling of two absorbing boundary conditions for 2D time-domain simulations of free surface gravity waves. J Comp Phys 1996;26:139-51.

[2] Fryer GJ, Watts P, Pratson LF, Gardner JV. The tsunami of 1 April 1946: a landslide in the upper forearc, Prediction of underwater landslide hazards. Rotterdam: Balkema Press, 2001.

[3] Grilli ST, Guyenne P, Dias F. Modeling of overturning waves over arbitrary bottom in a 3D numerical wave tank. Proceedings of the 10th International Offshore and Polar Engineering Conference, Seattle, USA, vol. III; 2000. p. 221-8.

[4] Grilli ST, Guyenne P, Dias F. A fully nonlinear model for threedimensional overturning waves over arbitrary bottom. Int $\mathbf{J}$ Numer Methods Fluids 2001;35(7):829-67.

[5] Grilli ST, Horrillo J. Numerical generation and absorption of fully nonlinear periodic waves. ASME J Engng Mech 1997;123(10):10609.

[6] Grilli ST, Svendsen IA. Corner problems and global accuracy in the boundary element solution of nonlinear wave flows. Engng Anal Bound Elem 1990;7(4):178-95.

[7] Grilli ST, Skourup J, Svendsen IA. The modeling of highly nonlinear waves: a step toward the numerical wave tank. In: Brebbia CA (editor), Proceedings of the 10th International Conference on 
Boundary Elements, Southampton, England, vol. 1. Berlin: Springer, Computational Mechanics Publication; 1988. p. 549-64.

[8] Grilli ST, Watts P. Modeling of waves generated by a moving submerged body. Applications to underwater landslides. Engng Anal Bound Elem 1999;23:645-56.

[9] Kim CH, Clément AH, Tanizawa K. Recent research and development of numerical wave tank-a review. Int J Offshore Polar Engng 1999;9(4):241-56.

[10] Murty TS. Submarine slide-generated water waves in Kitimat Inlet, British Columbia. J Geophys Res 1979;84(C12):7777-9.

[11] Tanaka M. The stability of solitary waves. Phys Fluids 1986;29(3):650-5.

[12] Tappin DR, Matsumoto T, Shipboard Scientists. Offshore surveys identify sediment slump as likely cause of devastating Papua New Guinea tsunami 1998. Eos 1999;80(30):329.

[13] Tappin DR, Watts P, McMurtry GM, Lafoy Y, Matsumoto T. The Sissano, Papua New Guinea tsunami of July 1998—offshore evidence on the source mechanism. Mar Geol 2001;175:1-23.
[14] Vogelmann S. Sensitivity study of numerical simulations of tsunami generated by submarine slope failure. MS Thesis, University of Rhode Island, Narragansett, RI, 2001.

[15] Watts P. Water waves generated by underwater landslides. PhD Thesis, California Institute of Technology, Pasadena, CA, 1997.

[16] Watts P. Wavemaker curves for tsunamis generated by underwater landslides. ASCE J Wtrwy Port Coast Oc Engng 1998;124(3):12737.

[17] Watts P. Tsunami features of solid block underwater landslides. ASCE J Wtrwy Port Coast Oc Engng 2000;126(3):144-52.

[18] Watts P, Imamura F, Grilli ST. Comparing model simulations of three benchmark tsunami generation cases. J Sci Tsunami Hazards 2000;18(2):107-23.

[19] Watts P, Grilli ST. Tsunami generation by submarine mass failure. Part I: Wavemaker models. Submitted for publication.

[20] Watts P, Grilli ST, Bengston A. Experimental validation of landslide tsunami simulation in a $3 \mathrm{D}$ numerical wave tank. In preparation. 